\title{
Plant genetic resources for improving stress tolerance - examples for cereals
}

\author{
Börner A. \\ Leibniz. Institute of Plant Genetics and Crop Plant Research (IPK), Gatersleben, Germany \\ email:boerner@ipk-gatersleben.de
}

Plant genetic resources for food and agriculture (PGRFA) play a major role for global food security. The most significant and widespread mean of conserving PGRFA is ex situ conservation. World-wide 7.4 million accessions are stored in about 1,750 ex situ genebanks. Plant ex situ genebank collections comprise seed genebanks, field genebanks as well as in vitro and cryo collections. Species whose seed can be dried, without damage, down to low moisture contents can be conserved in specially designed cold stores. Such "orthodox" seeds can be expected to maintain a high level of vigour and viability for decades. Field genebanks, in vitro and cryo storage are used primarily for species which are either vegetatively propagated or which have non-orthodox seeds that cannot be dried and stored for long periods. With a total inventory of 150,000 accessions from 3,212 plant species and 776 genera, the 'Federal ex situ Genebank of Germany' in Gatersleben holds one of the most comprehensive collections worldwide. It comprises wild and primitive forms, landraces as well as old and more recent cultivars of mainly cereals but also other crops. Starting in the 1920's material was accumulated systematically. Seed storage is managed in large cold chambers at $-18^{\circ} \mathrm{C}$. Seeds are kept in glass jars, covered with bags containing silica gel (active collection) and in aluminum bags under vacuum (base collection). The maintenance of the collection requires regeneration. Each year between 8 and 10 Thousand accessions are grown either in the field or in glasshouses. The collection has been widely characterized and evaluated. In the cereals, mainly wheat and barley, a number of bi-parental mapping populations and association mapping panels have been established to allow for the genetic analysis of various traits. The current focus covers resistance/tolerance to a number of biotic and abiotic stresses, in particular drought. 